\title{
A Comparative Study of Postural Stability in Subject with Hallux Valgus and Flat Feet: A Cross-Sectional Study
}

\author{
Rathod HP1*, Katherawala $\mathrm{S}^{2}$ Memon $\mathrm{A}^{1}$ and Lakhani N1 \\ ${ }^{1}$ Shrimad Rajchandra college of physiotherapy, Uka Tarsadia University, India \\ ${ }^{2}$ Shree Bhartimaiya College of optometry and Physiotherapy, Veer Narmad South \\ Gujarat University, India
}

\section{Research Article \\ Volume 1 Issue 2}

Received Date: September 24, 2018

Published Date: November 10, 2018

*Corresponding author: Heena Pranavsinh Rathod, Shrimad Rajchandra college of physiotherapy, Uka Tarsadia University, Maliba Campus,Bardoli, Surat- 394350, Gujarat India, Tel: +91 8980676644; Email: heena.solanki@utu.ac.in

\section{Abstract}

Background: Hallux valgus (HV) and Flat Foot (FF) are common conditions, affecting adults between 18-25 years of age. Today's styles of women's footwear encompass two key design features, an elevated heel and a constrictive toe box. Shoes with a narrow toe box increase pressures on the medial side of the foot and between the toes. Early shoe wearing in children impairs the development of longitudinal arches. Over time, these changes may contribute to the development of foot pain and deformity and may affect postural stability.

Aim and Objective: To assess and compare postural stability in subjects with Hallux Valgus and flat feet by using sense move.

Materials and Methods: Total 40 females were assessed and compared postural stability by using sense move. In which Group A included those with Hallux Valgus ( $N=20)$ and Group B included those with Flat foot deformity (N=20). Postural Stability was assessed and compared in centre and off-centre directions. Unpaired t-test was carried out for analysis of outcomes.

Results: The result of the study is, the Front and Back direction of postural stability in subject with Flat foot is more affected compared to the subject with hallux valgus and right and left side of postural stability in subject with hallux valgus is more affected compared to the subject with the Flat Foot.

Conclusion: The study concludes that the subjects with the hallux valgus were showing more affected postural stability in mediolateral (Left and Right) direction compare to subjects with FF, subjects with the Flat foot were showing more postural stability affected in Anteroposterior (Front and Back) direction Compare to subjects with HV.

Keywords: Hallux Valgus; Flat Foot; Postural Stability; Sensamove 


\section{Annals of Physiotherapy \& Occupational Therapy}

Abbreviations: HV: Hallux valgus; FF: Flat Foot; AP: Anterior-Posterior; COP: Center of Pressure; FP: Force Platform.

\section{Introduction}

Postural control under static conditions is usually called "postural steadiness," whereas the dynamic postural response to applied or volitional perturbations is called "postural stability" [1]. Different pathological foot conditions have different effect on postural control, among them Hallux valgus (HV) is a one of the common condition, affecting $23 \%$ of adults between $18-65$ years [2].

The woman's foot is wider in the forefoot and has shorter arch length, with the metatarsals shorter in comparison to man's foot. Today's styles of women's footwear encompass two key design features, an elevated heel and a constrictive toe box. Shoes with a narrow toe box increase pressures on the medial side of the foot and between the toes [3]. Over time, these changes may contribute to the development of foot pain and deformity.

Plantar foot loading is shifted medially and especially to the second and third metatarsal heads in the foot with pathological hallux valgus. Although sagittal plane motions are not affected, hind foot eversion is significantly larger in the terminal stance in the pathological HV group, indicating an unstable foot. These alterations can affect postural stability and balance, single-limb postural stability and proprioception are key components of the functional status of the lower extremity.

Impaired postural stability is a risk factor for ankle and knee injuries. Factors affecting postural stability should be well defined to prevent injuries. It is known that symptoms of the hallux valgus deformity are -usually not severe early but the condition can become painful later and affect functional status in adults and older people [2]. However we believe that a pathological HV angle may affect postural stability even if the patient does not experience pain [4].

The arches are considered to play functionally significant roles by supporting body weight and reducing the impact of the body during running and walking [5] During standing, the arch is thought to not only support weight but also contribute to dynamic postural control and equilibrium maintenance; however, these functions have not been fully elucidated.
Cobb, et al. reported that the subjects with bilateral fore foot varus greater than or equal to $7^{\circ}$ had a significantly poorer anterior-posterior (AP) postural stability (standard deviation of the AP ground reaction force) than the group with forefoot varus less than $7^{\circ}$ for a 5 -second, barefoot, single-leg-stance balance task with eyes close [6].

The working methodology in our study is focused on using the Balance Miniboard manufactured by Sensamove in order to measure the level of static, dynamic balance and proprioceptive memory. In previous studies laboratory-based assessment using measures of center of pressure (COP) recorded from a force platform (FP) which is considered the gold standard measure of balance have identified important outcome measures which are too subtle to detect using a subjective scale [7]. Using a FP to assess standing balance provides useful information, however they are often expensive, difficult to setup and cumbersome to transport and therefore this form of balance assessment is often not feasible in a clinical setting.

Consequently, subjective assessment tools which do not require specialized equipment, such as the Berg Balance Scale, are commonly used and have also been shown to provide valuable information. While these protocols are more clinically applicable, they suffer from limitations including ceiling effects and a limited precision to detect small changes in performance [7].

In addition, previous research indicates that the relationship between scores on subjective tests and measures of COP displacement is only moderate, and that a combination of the two protocols may provide important information which cannot be obtained by either subjective or quantitative assessment alone [7]. The sensamove mini balance board has similar characteristics to a laboratory grade FP, which are used to assess force distribution and the resultant movements in COP.

It was hypothesized that there is significant difference in postural stability in subject with Hallux valgus and flat feet using sensamove mini balance board.

The present study is designed to compare postural stability in subject with Hallux valgus and flat feet by using sensamove and also to assess the effect of Hallux valgus on postural stability and to assess the effect of flat feet on postural stability. 


\section{Methodology}

The study was conducted including 40 subjects (only females) with hallux valgus and flat feet selected through convenient sampling technique from Uka Tarsadia University. The subjects were selected on the basis of the age group between 18-25 years, great toe adduction is $>10$ degree, flat foot with ND of $>10 \mathrm{~mm}$ and subjects with any type of foot and ankle injury, any Neurological or systemic disorder, musculoskeletal pain, any type of Lower extremity deformity and vestibular impairment.

The study was cleared by the ethical committee of Shrimad Rajchandra college pf physiotherapy.

20 subjects were evaluated with a goniometer for the $\mathrm{HV}$ angle, and were selected if their angle was found greater than 10 degrees and other 20 subjects were evaluated for flat foot with the help of vernier calliper, flat foot found ND of $\geq 10 \mathrm{~mm}$ were selected. By both measurements total 40 subjects were selected.
A brief explanation about the study was given to them and a written consent form was signed prior to participation in the study. 40 subjects were divided in to two groups by convenient sampling. Group (a) who had Hallux valgus, and Group (b) who had flat feet.

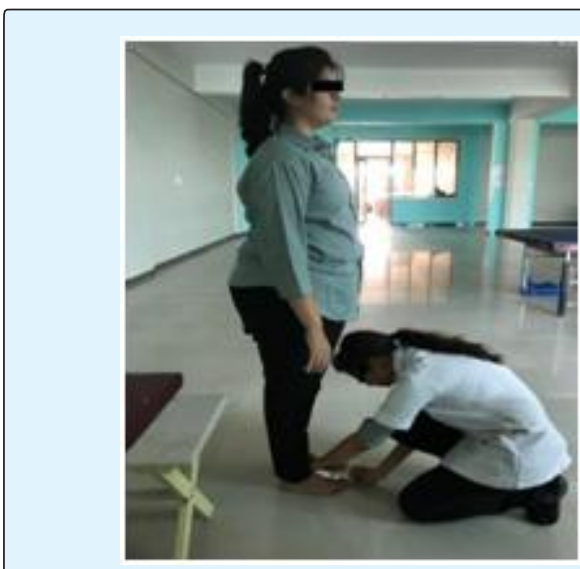

Figure 1: Measurement of Hallux Valgus.

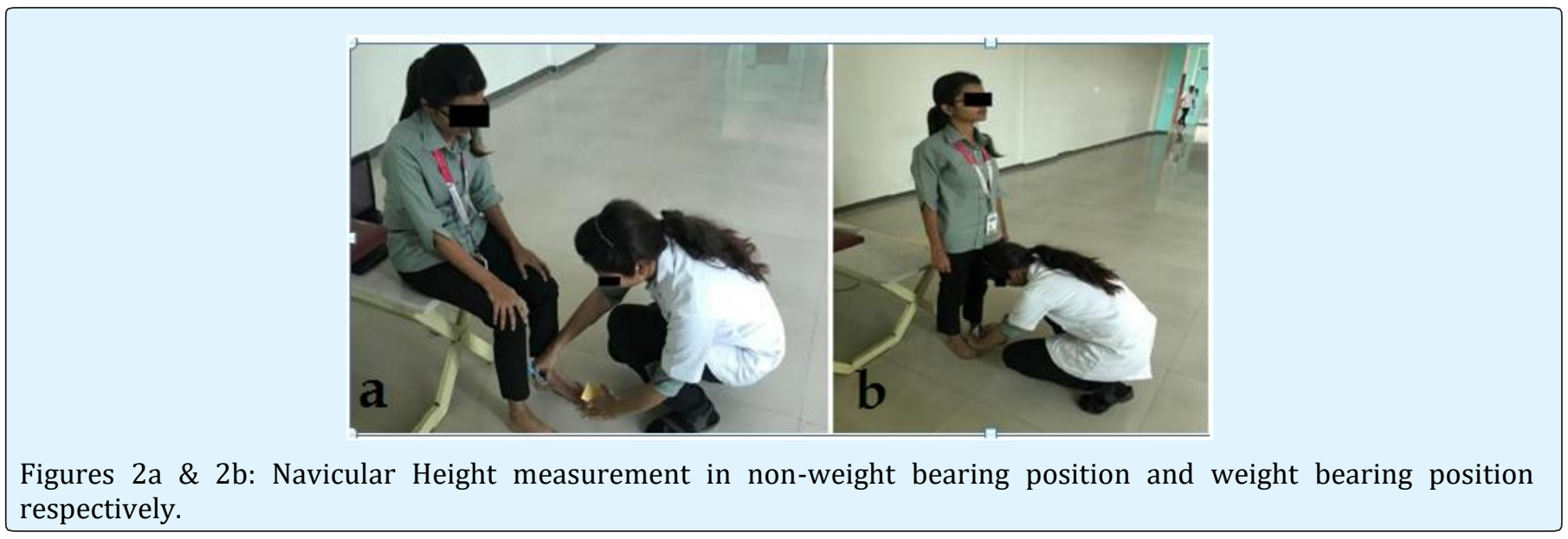

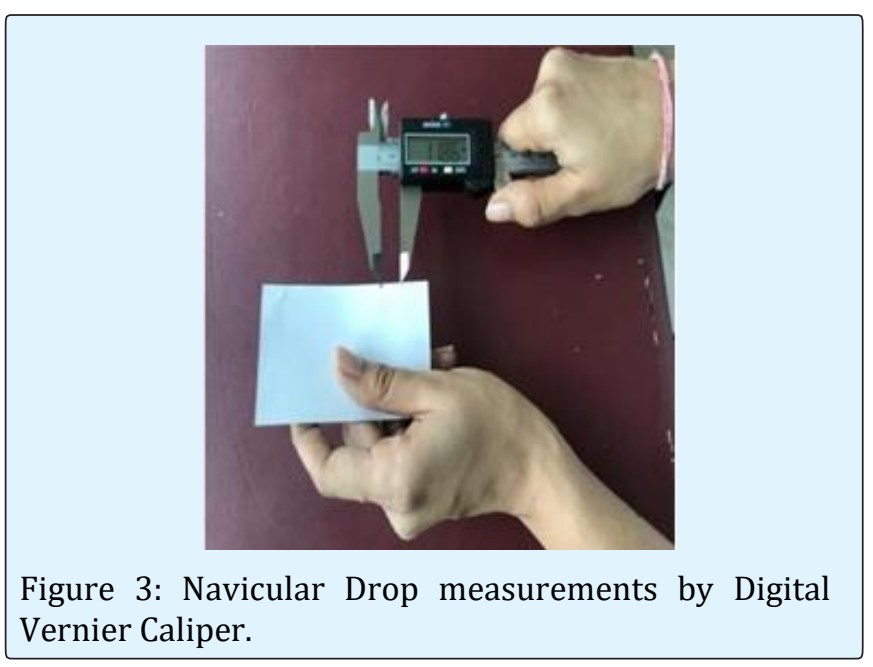

Rathod HP, et al. A Comparative Study of Postural Stability in Subject with Hallux Valgus and Flat Feet: A Cross-Sectional Study. Ann Physiother Occup Ther 2018, 1(2): 000108.
Procedure to assess postural stability: Static stability consist of 5 positions that is center and off-center positions at $80 \%$ of the measured maximum Rom front, back, left and right. The subjects were first explained regarding the procedure.

The subject was asked to start from neutral position (red dot in center of screen), by standing on sensamove mini board, and click 'start exercise' button. The subject was asked to maintain this posture for six seconds as stable as possible by keeping the red dot as close to the center as possible. A stop watch on the screen was showed, marking the countdown of the six seconds. 
After six seconds when the measurements were ready, then click 'finish exercise' button. By pressing the results tab at the right of the screen, intermediate results can be viewed and were recorded. Click on ok and start next measure. Now measurement for the four directions front, back, left and right.

Asked subject to stand on sensamove mini board, an orange target disk appeared at $80 \%$ of the maximum rom in the direction of the specific directional exercise. Start from neutral position (red dot in the center of the screen), click 'start exercise' button and move to the orange target disk. Maintain posture at this position for six seconds as the screen was 2 . After six seconds the measurements were ready. Click 'finish exercise'. Click on the next for next directional. The same procedure was repeated for the rest 3 directions and the results were collected. The smaller the value, the better the static balance ability showed for the countdown of the six seconds.

\section{Results}

Descriptive statistics including mean and standard deviation were analyzed. Unpaired t-test was used for comparing means between all the two groups. Paired ttest was used to compare the difference within the groups. Results were found to be significant at $\mathrm{p}<0.05$ and confidence interval was set at $95 \%$. All statistical analysis was performed using Microsoft Excel.

Table1 shows that the mean value of age in subjects with hallux valgus is 20.1 where mean value of right leg subjects with hallux valgus is 13.25 and mean value of left leg subjects with hallux valgus is 14.2.

\begin{tabular}{|c|c|c|c|c|c|}
\hline & Age & Height & Weight & Right & Left \\
\hline Mean & 20.1 & 1.586 & 52.5 & 13.3 & 14.2 \\
\hline Median & 20 & 1.575 & 49.5 & 12 & 12 \\
\hline $\begin{array}{c}\text { Standard } \\
\text { Deviation }\end{array}$ & 1.07 & 0.062 & 13.41 & 3.45 & 4.88 \\
\hline Minimum & 18 & 1.48 & 37 & 9 & 8 \\
\hline Maximum & 22 & 1.72 & 86 & 22 & 28 \\
\hline
\end{tabular}

Table 1: Baseline description statistics of Age, Height, Weight, Right leg and Left leg of Hallux Valgus.

Table2 shows that mean value of age in subjects with flat foot is 19.75 where mean value of right leg subjects with flat foot is 10.654 and mean value of left leg subjects with flat foot is 9.911 .

\begin{tabular}{|c|c|c|c|c|c|}
\hline & Age & Height & Weight & Right & Left \\
\hline Mean & 19.75 & 1.575 & 54.45 & 10.65 & 9.911 \\
\hline Median & 20 & 1.575 & 55 & 10.83 & 10.26 \\
\hline $\begin{array}{c}\text { Standard } \\
\text { Deviation }\end{array}$ & 1.118 & 0.051 & 10.75 & 2.29 & 2.54 \\
\hline Minimum & 18 & 1.49 & 37 & 4.39 & 4.6 \\
\hline Maximum & 22 & 1.67 & 74 & 14.47 & 14.62 \\
\hline
\end{tabular}

Table 2: Baseline description statistics of Age, Height, Weight, Right leg and Left leg of Flat Foot.

Graph-1 shows that the Front and Back direction postural stability in subject with Flat foot is more affected than the subject with hallux valgus and Right and left direction of postural stability in subject with hallux valgus is more affect than the subject with the Flat Foot.

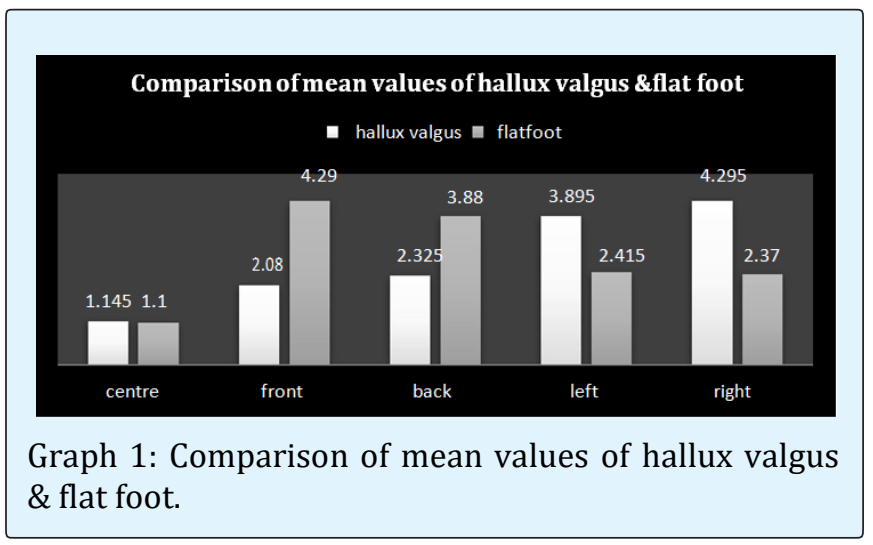

Table 3 represents that the significance of the $P$ value is 0.603 where $T$ value is 0.524 . So the $P$ value is suggesting that there is no significant difference of postural stability in subject with hallux valgus and flat foot.

\begin{tabular}{|c|c|c|c|c|c|}
\hline & Variation & $\begin{array}{c}\text { Mean } \\
\text { Difference }\end{array}$ & $\begin{array}{c}\text { Degree Of } \\
\text { Freedom }\end{array}$ & P value & T value \\
\cline { 1 - 2 } $\begin{array}{c}\text { Hallux } \\
\text { Valgus }\end{array}$ & 0.060 & 0.045 & 37 & 0.603 & 0.524 \\
\cline { 1 - 1 } $\begin{array}{c}\text { Flat } \\
\text { Foot }\end{array}$ & 0.086 & & & & \\
\hline
\end{tabular}

Table 3: of Variation, Mean Difference, Degree of Freedom, $\mathrm{P}$ value and $\mathrm{T}$ value for Centre Position.

Table 4 represents that the $P$ value is 0.00 and $T$ value is -2.9. So the $P$ value is suggesting that there is a significant difference of postural stability in subject with hallux valgus and flat foot. 


\begin{tabular}{|c|c|c|c|c|c|}
\hline & Variation & $\begin{array}{c}\text { Mean } \\
\text { Difference }\end{array}$ & $\begin{array}{c}\text { Degree Of } \\
\text { Freedom }\end{array}$ & P value T value \\
\cline { 1 - 2 } $\begin{array}{c}\text { Hallux } \\
\text { Valgus }\end{array}$ & 0.731 & 2.21 & 22 & 0.008 & -2.905 \\
\cline { 1 - 2 } $\begin{array}{c}\text { Flat } \\
\text { Foot }\end{array}$ & 10.837 & & & & \\
\hline
\end{tabular}

Table 4: Table of Variation, Mean Difference, Degree of Freedom, P value and T value for Front Direction.

Table 5 represents that the $P$ value is 0.00 and $T$ value is -5.05 . So the $P$ value is suggesting that there is a significant difference of postural stability in subject with hallux valgus and flat foot.

\begin{tabular}{|c|c|c|c|c|c|}
\hline & Variation & \begin{tabular}{|c|} 
Mean \\
Differences \\
\end{tabular} & \begin{tabular}{|l|} 
Degree of \\
Freedom \\
\end{tabular} & P value & $\begin{array}{c}\mathrm{T} \\
\text { value }\end{array}$ \\
\hline \begin{tabular}{|l|} 
Hallux \\
Valgus
\end{tabular} & 0.694 & \multirow{2}{*}{1.555} & \multirow{2}{*}{36} & \multirow{2}{*}{0.00} & \multirow{2}{*}{-5.059} \\
\hline \begin{tabular}{|c|} 
Flat \\
Foot
\end{tabular} & 1.194 & & & & \\
\hline
\end{tabular}

Table 5: Table of Variation, Mean Difference, Degree of Freedom, $\mathrm{P}$ value and $\mathrm{T}$ value for Back Direction.

Table 6 represents that the $P$ value is 0.00 and $T$ value is 4.44. So the $\mathrm{P}$ value is suggesting that there is a significant difference of postural stability in subject with hallux valgus and flat foot.

\begin{tabular}{|c|c|c|c|c|c|}
\hline & Variation & $\begin{array}{c}\text { Mean } \\
\text { Differences }\end{array}$ & $\begin{array}{c}\text { Degree Of } \\
\text { Freedom }\end{array}$ & P valueT value \\
\cline { 1 - 2 } $\begin{array}{c}\text { Hallux } \\
\text { Valgus }\end{array}$ & 1.392 & 1.48 & 36 & 0.00 & 4.447 \\
\cline { 1 - 4 } $\begin{array}{c}\text { Flat } \\
\text { Foot }\end{array}$ & 0.822 & & & & \\
\hline
\end{tabular}

Table 6: Table of Variation, Mean Difference, Degree of Freedom, $\mathrm{P}$ value and $\mathrm{T}$ value for Left Direction.

Table 7 represents that the $P$ value is 0.00 and $T$ value is 5.43. So the $P$ value is suggesting that there is a significant difference of postural stability in subject with hallux valgus and flat foot.

\begin{tabular}{|c|c|c|c|c|c|}
\hline & Variation & $\begin{array}{c}\text { Mean } \\
\text { Differences }\end{array}$ & $\begin{array}{l}\text { Degree Of } \\
\text { Freedom }\end{array}$ & $P$ value & $e$ \\
\hline $\begin{array}{l}\text { Hallux } \\
\text { Valgus }\end{array}$ & 1.923 & \multirow{2}{*}{1.925} & \multirow{2}{*}{30} & \multirow{2}{*}{0.00} & \multirow{2}{*}{5.432} \\
\hline $\begin{array}{l}\text { Flat } \\
\text { Foot }\end{array}$ & 0.587 & & & & \\
\hline
\end{tabular}

Table 7: Table of Variation, Mean Difference, Degree of Freedom, P value and T value for Right Direction.
- The mean age of subjects with hallux valgus is 20.1(S.D \pm 1.07$)$. The subjects with hallux valgus, mean value of right leg is $13.25(\mathrm{~S} . \mathrm{D} \pm 3.44)$ and the subjects with hallux valgus, mean value of left leg is 14.2(S.D \pm 4.88$)$.

- The mean age of subjects with flat foot is 19.75(S.D \pm 19.75$)$. The subjects with flat foot, mean value of right and left leg respectively is 10.654(S.D \pm 10.65$)$ and 9.911(S.D \pm 9.91$)$.

- The Front and Back direction of postural stability in subject with Flat foot is more affected compared to the subject with hallux valgus and Right and left direction of postural stability in subject with hallux valgus is more affected compared to the subject with the Flat Foot.

- $\mathrm{P}$ value is suggesting that there is no significant difference of postural stability in subject with hallux valgus and flat foot in centre position.

- $P$ value is suggesting that there is a significant difference in Front, back, left and right direction in subjects with the hallux valgus and flat foot. $(p<0.05)$

\section{Discussion}

The aim of our study was to assess the postural stability in subject with the hallux valgus and flat foot and to compare the postural stability in subjects between the two conditions. Our study focused on the effect of hallux valgus and flat foot on postural stability to show whether the asymptomatic subjects with hallux valgus deformity and flat foot deformity affects postural stability.

The proprioceptive feedback mechanism is necessary to ensure postural control, sensory input from the skin, muscles, joints and ligaments is essential for proprioception. Postural control is a key factor in preventing injuries and falls, and requires foot sense of motion and the ability to make alterations. A foot abnormality may alter sensory input from the plantar surface and joints. Dynamic factors such as ankle instability have been shown to cause poor postural control. Structural foot abnormalities have also been investigated and it is generally accepted that the type of foot abnormality affects postural stability [2] .

The findings of our study is Hallux valgus deformity affect the postural stability in Mediolateral direction in correlation to the study of Wen J, et al. shows that the mild hallux valgus angle negatively affected single-limb balance in their study. Subjects with a pathological mild $\mathrm{HV}$ angle had decreased ability to maintain their stability compared with normal subjects and mediolateral stability 


\section{Annals of Physiotherapy \& Occupational Therapy}

scores showed greater stability scores in these patients compared to normal feet. Inability to maintain mediolateral stability may be influenced by the altered loading strategies of the fore foot [2]. Nix, et al. found a moderate hallux valgus angle (mean $\pm \mathrm{SD}=29.1 \pm 7.8$ ) to affect mediolateral postural sway negatively during bilateral standing in adults (mean age $=44.5$ years).

Menz et al, found that a moderate or severe HV angle affects the maximum balance range and walking speed but not the sway area in older adults [2]. Pronation of the first metatarsal and an imbalance of intrinsic muscles have been reported with the hallux valgus deformity and could also affect the segmental stability of the foot. Articular mechanoreceptors and muscle receptors have an important role in providing sensory input for segmental and postural control, proprioceptive information from misaligned joints and muscles might have a negative effect on postural control. These transitions might have an effect on single-limb stability of patients with an increased HV angle and it explains increased $\mathrm{HV}$ angle was seen with worse postural stability scores [2]. There are many parameters that affect postural stability. Increased knee eccentric extensor strength has been shown to reduce postural sway. Reduced joint position sense, tactile sensitivity and vibration sense in the lower extremity, reduced quadriceps and ankle dorsiflexion strength, and slow reaction times are all associated with impaired postural stability [2]. However, we only investigated the effect of the HV angle and FF on postural stability in this study.

The result of our study is that, that the effect of Flat foot deformity affect postural stability in Anteroposterior direction(Front and Back) where the previous study, mahshid S, et al. have reported a flatter foot resulted in increased anteroposterior sway [8]. Cobb, et al. reported that the subjects in the pronated group had poorer standing postural control than subjects in the neutral group in terms of a greater normalized center-of pressure standard deviation and maximum displacement in the AP direction. The finding states that the subjects with Flat foot had a significantly greater normalized center postural stability standard deviation and maximum displacement in the AP direction than the subjects with $\mathrm{HV}$ in the current study is in agreement with the results reported by Cobb et al [6]. Cobb, et al. hypothesized that, the subjects with a pronated foot structure would have poorer standing postural control than subjects with neutral feet, because of reduced stability within the foot joints. Subjects in the pronated foot group, however, might also have had some advantage from the increased
ML dimension of the base of support that coincides with foot pronation [9-11].

The possible deleterious influence on postural control caused by passive instability in the joints of the foot, therefore, might be attenuated somewhat in the ML direction by the positive influence of an enlarged base of support. This may explain why the pronated foot group had a significantly greater normalized center of pressure standard deviation and maximum displacement than the neutral group in the AP direction and not in the ML direction(6). The results of our study are Hallux valgus deformity affect the postural stability in Mediolateral direction (Right and Left) where the Flat Foot deformity affects the postural stability in Anteroposterior (Front and Back) direction [12-15].

\section{Conclusion}

The study concluded that the subjects with the hallux valgus they were showing more affected postural stability in mediolateral (Left and Right) direction compare to subjects with FF and subjects with the Flat foot as they were showing more postural stability affected in Anteroposterior (Front and Back) direction Compare to subjects with HV.

\section{Limitations of the Study}

Different category of hallux valgus was not included like moderate and severe HV. Study included only females.

\section{References}

1. Chaudhry H, Findley T, Quigley KS, Bukiet B, Ji Z, et al. (2004) Measures of postural stability. Journal of Rehabilitation Research and Development 41(5): 713-720.

2. Çınar-Medeni O, Atalay Guzel N, Basar S (2016) Hallux valgus angle affects single-limb postural stability in asymptomatic subjects. Journal of Backand Musculoskeletal Rehabilitation 29(1): 117-121.

3. Menz HB, Roddy E, Marshall M, Thomas MJ, Rathod T, et al. (2016) Epidemiology of Shoe Wearing Patterns Over Time in Older Women: Associations With Foot Pain and Hallux Valgus. Journals of Gerontology: Medical Sciences 71(12): 1682-1687.

4. Ashok A., Manoj Mohan K, Achleshwar R Gandotra (2017) Prevalence of Flexible Flat Foot in Adults: A 


\section{Annals of Physiotherapy \& Occupational Therapy}

Cross sectional Study. Journal of Clinical and Diagnostic Research 11(6): AC17-AC20.

5. Emi A, Kanako N, Yumi I, Mitsuru S, Shuichi I, et al. (2014) Effects of Foot Arch Structure on Postural Stability. Clinical Research on Foot Ankle 2(2): 1-5

6. Tsai LC, Yu B, Mercer VS, Gross MT (2015) Comparison of Different Structural Foot Types for Measures of Standing Postural Control. Journal of Orthopedic \& Sports Physical Therap 36(12): 942953.

7. Clark RA, Bryant AL, Pua Y, McCrory P, Bennell K, et al. (2010) Validity and Reliability of the Nintendo Wii Balance Board for assessment of Standing Balance. Gait and Posture 31(3): .307-310.

8. Mahshid Saghazadeh, Kenji Tsunoda, Tomohiro Okura (2014) Foot arch height and rigidity index associated with balance and postural sway in elderly women using a 3D foot scanner. The Foot and Ankle Online Journal 7(4): 1

9. Robert W, Katarzyna W, Tomasz R, Marcin B (2015) The Assessment of Postural Stability of Women with Hallux valgus. Medical Review 14(1): 48-60.
10. Esther H Kim, Howard J Hillstom, Benjamin P (2005) Heilman Effect of Arch Height and Accomodation on Postural Stability.

11. Kevin D, Ivan B, Kaat D, Matricali GA (2010) The Impact of Hallux valgus on Foot Kinematics: A CrossSectional, Comparative Study. Gait and Posture 32(1): 102-106.

12. Hurn SE, Vicenzino B, Smith MD (2015) Functional Impairments Characterizing Mild, Moderate and Sever Hallux valgus. Arthritis care and Research 67(1): 80-88.

13. Razieh T, Mohammad T Karimi, Behnaz S, Francis F (2014) Evaluation of Standing Stability in individual with Flatfeet. SAGE Journal 8(3): 168-174.

14. Aryadoost H, Hosain Alizadeh M (2014) Comparison of Static and Dynamic Balance in Female Athletes with and without Hallux Valgus. International Journal of Sport Studies 4(12): 1473-1478.

15. Arani SJ, Jahanmahin M, Arani MA, Arani HH (2016) The effect of lower body skeletal disorders on dynamic and static balance in 12-14 years old girls. International Journal of Sport Studies 6(6): 391-394. 